\title{
Precise orbit determination of Haiyang-2 using satellite laser ranging
}

\author{
ZHAO Gang $^{1,2^{*}}$, ZHOU XuHua $^{1} \&$ WU Bin ${ }^{1 *}$ \\ ${ }^{1}$ Shanghai Astronomical Observatory, Chinese Academy of Sciences, Shanghai 200030, China; \\ ${ }^{2}$ University of Chinese Academy of Sciences, Beijing 100049, China
}

Received August 6, 2012; accepted September 23, 2012; published online November 27, 2012

\begin{abstract}
With the successful launch and official commissioning of China's first dynamic ocean environment satellite Haiyang-2 (HY-2), China's capabilities for oceanic environment monitoring and oceanic resource detecting have been further improved and enhanced. Precise tracking and orbit determination are not only key technical concerns in the ocean dynamic environment satellite project but also necessary conditions for carrying out related oceanic science research using observational data obtained using spaceborne instruments including radar altimeter. In this study, the current available status of international satellite laser ranging (SLR) monitoring on HY-2 was introduced. Six-months of SLR data from HY-2 were processed to obtain precise satellite orbit information using the dynamic orbit determination method. We carried out a detailed assessment of the SLR orbit accuracy by internal evaluation, comparisons with the orbit derived by the French Doppler orbitography and radio-positioning integrated by satellite (DORIS) system, and station-satellite distance validation. These assessments indicate that the three-dimensional orbital accuracy of HY-2 is about $12.5 \mathrm{~cm}$, and the radial accuracy is better than $3 \mathrm{~cm}$. It provides a good example of the application of international SLR monitoring and precise orbit determination in China's earth observation satellite project.
\end{abstract}

Haiyang-2, satellite laser ranging, precise orbit determination

Citation: Zhao G, Zhou X H, Wu B. Precise orbit determination of Haiyang-2 using satellite laser ranging. Chin Sci Bull, 2013, 58: 589-597, doi: 10.1007/ s11434-012-5564-6

China's first ocean dynamic environment satellite, Haiyang-2 (HY-2), was successfully launched on August 16, 2011, and officially commissioned by the State Oceanic Administration of China on March 2, 2012 (www.soa. gov.cn). HY-2 is the third Chinese ocean satellite following HY-1A and HY-1B [1] with a designed lifetime of more than three years. Owing to the integration of active and passive microwave remote sensors, HY-2 provides all-weather, continuous and global detecting capabilities. The spaceborne payloads include radar altimeter, microwave scatterometer, microwave scanning radiometer, microwave calibrating radiometer and other detection instruments [2,3]. The National Satellite Ocean Application Service (NSOAS) of China has made use of its detection results to publish scientific information on, for example, atmospheric water

*Corresponding authors (email: zhaogang@shao.ac.cn; bwu@ shao.ac.cn) vapor content, sea surface windfield, sea surface temperature, sea surface height and significant wave height (www. nsoas.gov.cn).

HY-2 travels in a twilight, sun-synchronous near circular orbit, with an orbital inclination of $99.34^{\circ}$ and a local time of the descending node of 6:00 am. A two-phase orbit design was adopted. In the early stage, the orbit is recursive and frozen, with a repeat cycle of $14 \mathrm{~d}$, height of $971 \mathrm{~km}$, and orbital period of $104.46 \mathrm{~min}$, while in the later stage, the orbit is recursive, with a repeat cycle of 168 days, height of $973 \mathrm{~km}$, and orbital period of $104.50 \mathrm{~min}$. HY-2 uses three independent methods for orbit determination, global positioning system (GPS), Doppler orbitography and radio-positioning integrated by satellite (DORIS), and satellite laser ranging (SLR). These provide HY-2 with the highest precision orbit tracking and orbit determination capabilities among current Chinese remote sensing satellites. 
A high-precision orbit plays a fundamental role in exploiting measurements from various types of onboard instruments involved in assisting related oceanic scientific research. An important objective of HY-2 is to measure sea surface height, in which the orbital precision, especially its radial component, is the key factor in the application of data from the radar altimeter [4,5]. As an important satellite tracking and positioning technique, SLR derives the distance between a ground-based laser station and the satellite directly from the round-trip propagation time of a laser pulse, so its measurement is most sensitive to the satellite's radial position, and can provide efficient checks on satellite altimetry readings $[6,7]$. Besides its role in orbit determination, SLR data have been widely used in scientific studies. Scientists, including many Chinese researchers, have developed and will explore the functions of high-precision SLR observations in the fields of astrometry, astro-geodynamics and geophysics [8-11]. In this work, we summarize the current SLR observation on HY-2, derive its precise orbit by adopting dynamic orbit determination techniques, and assess the orbit precision in different ways including internal accuracy evaluation, comparison with the DORIS medium orbit ephemeris (MOE), and independent SLR station-satellite ranging validation.

\section{SLR tracking performance}

To achieve the goal of SLR observation, a hemispherical laser retro-reflector array (LRA) is positioned on the panel of the HY-2 satellite body facing the earth. The LRA is composed of nine circular Ag-coated quartz corner cubes, each with a radius $16.5 \mathrm{~mm}$ and height $26.2 \mathrm{~mm}$ [12]. Multiple orbit maneuvers are needed to maintain the proper operation of the onboard instruments and ensure the successful completion of mission objectives. After the maneuver at UTC 16:20, September 28, 2011, Changchun station in China achieved the first SLR tracking of HY-2 on October 2, 2011.

At present the international laser ranging service (ILRS) is responsible for the observation, data and product delivery of the global SLR tracking network [13]. HY-2 has been taken as the observational target [14] of continually-refined ILRS network $[13,15]$. Until the end of April, 2012, tracking data were obtained from 24 SLR stations. The locations, names and ID (identifiers) are shown in Figure 1. From the aspect of geographical distribution, there are more stations in the northern than in the southern hemisphere, and more in the eastern than in the western hemisphere. Part of the global ILRS network can implement daylight and/or high repetition-rate $(\mathrm{kHz})$ operations.

The data used in the process of precise orbit determination (POD) of SLR are normal point data. Each normal point is a compressed datum of the original tracking data from within a fixed period of time (called a time window) according to a properly sequenced algorithm, so it can represent all the information contained in the original data. The size of the time window is mainly determined by the

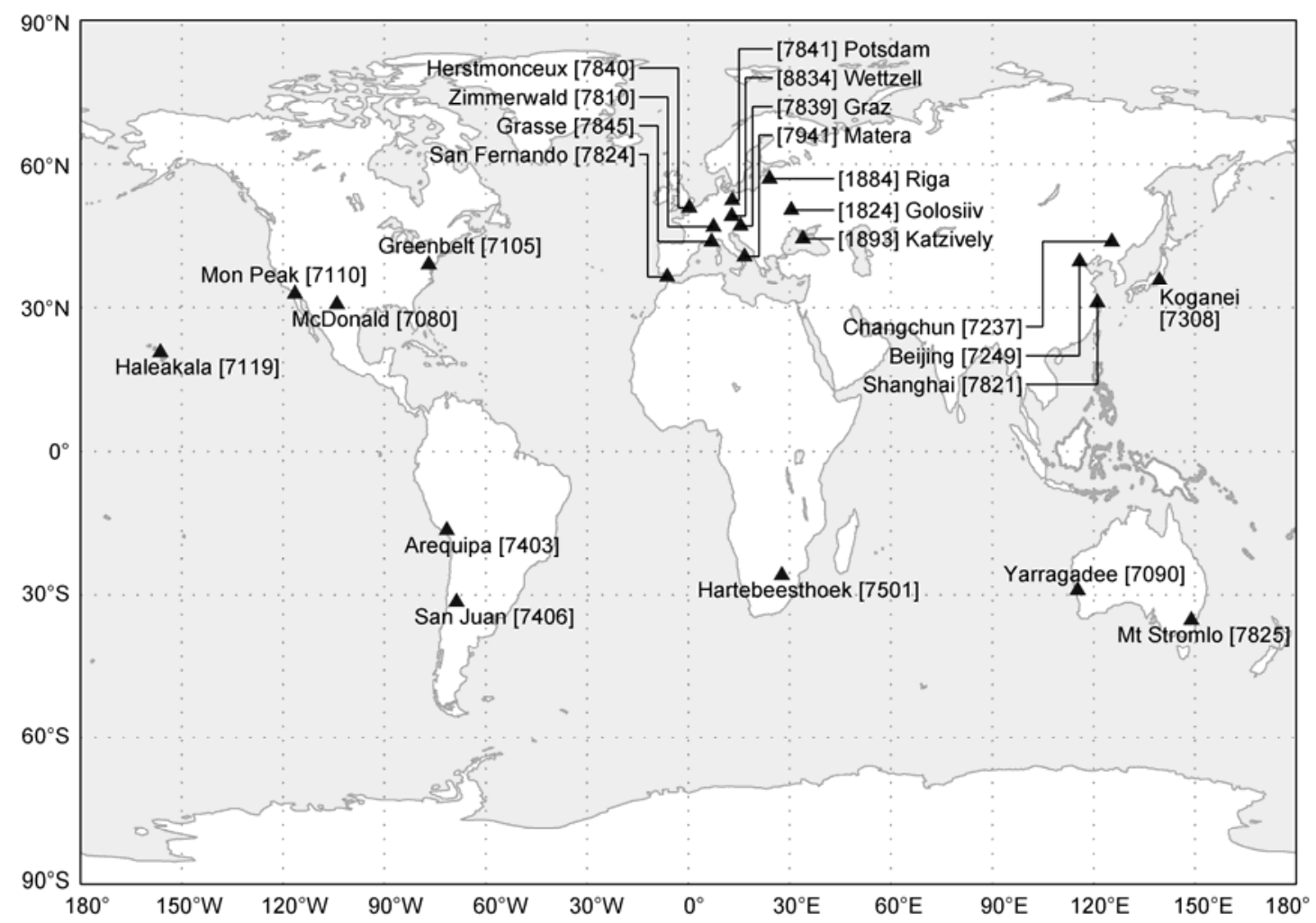

Figure 1 Geographic distribution of SLR stations which tracked HY-2. 
altitude of the satellite orbit, which is $30 \mathrm{~s}$ for HY-2.

During the seven months from October, 2011 to April, 2012, a total of 2255 passes were tracked and 21537 normal points were generated from the global ILRS observations on HY-2. The SLR tracking capability is significantly influenced by the weather conditions. With the disparity of the operational and meteorological status, there exist obvious differences between the quantities of observational data from the 24 SLR stations, as shown in Figure 2. The locations in the horizontal axis represent identifiers of stations, and the bar heights show passes (Figure 2(a)) and number of normal points (Figure 2(b)) at each station. The lightcolored bars represent the Chinese SLR network, including Changchun (ID 7237), Beijing (ID 7249), Shanghai (ID 7821), and San Juan (ID 7406). Gross observations of 340 passes and 2436 normal points were tracked by the Chinese SLR network, which take up more than $10 \%$ of the global volume.

Figure 3 describes the temporal distributions of global SLR tracking on HY-2, which are, from top to bottom, the number of tracking stations (Figure 3(a)), total passes (Figure 3(b)) and quantity of normal points (Figure 3(c)) per day. The $x$-coordinate is the time of the MJD (modified Julian Date). The vertical solid lines in the figure divide the time span by month, and the down arrows indicate when the maneuvers were performed, which was February 14, 2012 (UTC 03:05), February 24, 2012 (UTC 03:20) and April 20, 2012 (UTC 03:20). It can be seen that during these 212 days,
HY-2 was tracked by SLR every day except October 4, 2011. On average, there were 6 to 7 stations observed with 10 to 11 passes each day, and the daily mean of the normal points was about 102 .

\section{SLR POD strategy}

Using the normal point data of HY-2, and by applying statistical estimation techniques, the procedure for dynamic orbit determination can be carried out in which the precise orbit can be calculated, and at the same time, certain dynamic parameters can be estimated. The well-established perturbation force models and the properly selected scheme for parameter solution are the key factors in dynamic orbit determination [16].

All forces acting on a satellite body are perturbations except the central gravitational force. To take into account unmodeled or mismodeled errors and observational errors more efficiently, some selected dynamic parameters are usually treated as estimated parameters, and are solved together with the determination of the satellite orbit. The configuration of estimated parameters should not only make orbit accuracy keep high enough, but also make the correlation between each pair of estimated parameters as weak as possible, so as to guarantee the accuracies of the solutions to normal equations. The specific perturbation models and estimated parameters involved in the orbit determination are
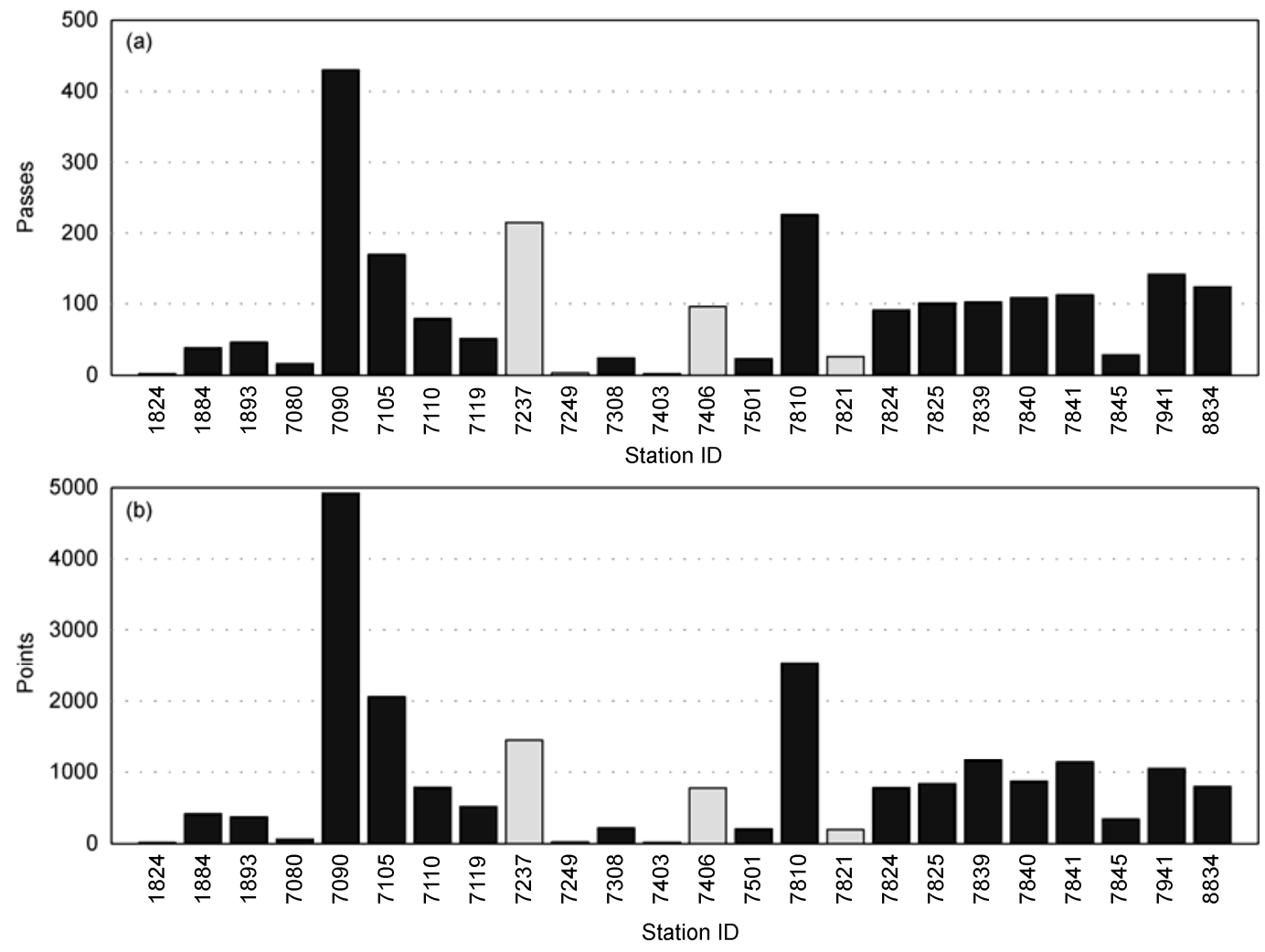

Figure 2 Numbers of passes (a) and normal points (b) at each SLR station. 


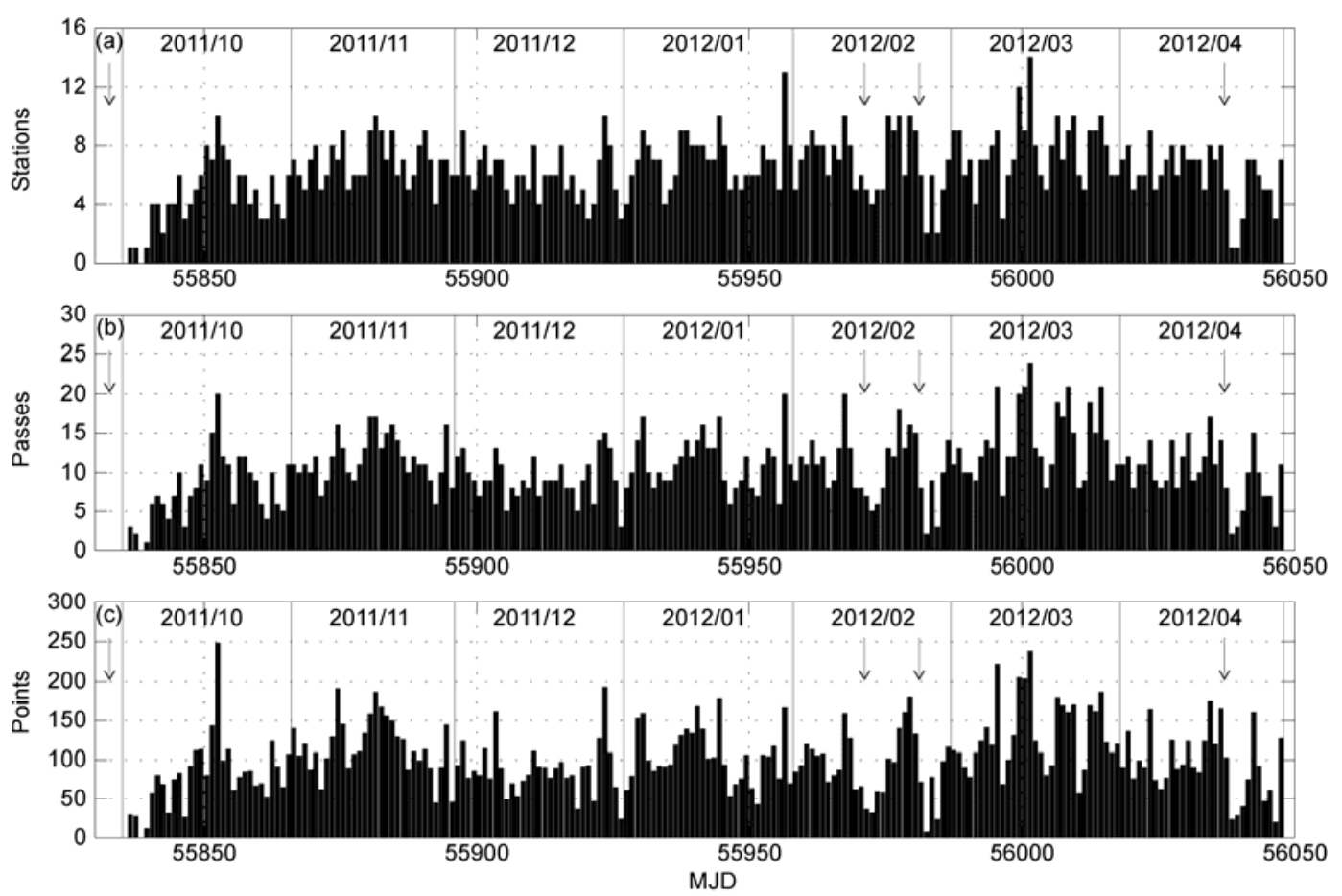

Figure 3 Number of stations, passes, and normal points in daily SLR tracking.

Table 1 Models and parameters used in orbit determination for HY-2

\begin{tabular}{lll}
\hline Perturbation & Description & Remarks \\
\hline Earth gravity & GGM02C model, 150×150 & Tapley et al. 2005 [17] \\
N-body perturbation & JPL DE 405 Ephemeris, including solar, lunar and planetary gravitations & Standish 1998 [18] \\
Atmospheric drag & DTM 94 model & Berger et al. 1998 [19] \\
Solar radiation pressure & Box-Wing model & Rim 1992 [20] \\
Solid earth tides & recommended model & IERS convention (1996) [21] \\
Ocean tides & CSR 4.0 model & Eanes and Schuler 1999 [22] \\
Relativistic perturbation & recommended model & IERS convention (2003) [23] \\
Periodic RTN perturbation & empirical force model & Colombo 1989 [24] \\
Estimated parameters & & \\
Initial state vector & 3-D position and velocity & estimated every 3 days \\
Drag coefficient & subarc estimation & estimated every 0.5/1.0 day (see text) \\
Solar radiation pressure coefficient & global estimation & estimated every 3 days \\
T, N empirical force coefficients & global estimation & estimated every 3 days \\
\hline
\end{tabular}

listed in Table 1. Considering the quantities of SLR tracking data obtained, conventionally an orbital arc length of 3 days is adopted.

The atmospheric drag, proportional to atmospheric density, is one of the most complicated perturbations acting on low-orbit satellites such as HY-2. The atmospheric density is closely related to solar and geomagnetic activities [25]. The 24th solar cycle, beginning in 2008, is now in its development stage [26,27], and thus occasionally the current solar radiation and earth geomagnetic field undergo dramatic changes. Because the error introduced in extreme conditions by the atmospheric model can reach up to $20 \%$
$[19,25]$, and the temporal distribution of HY-2 SLR tracking is uneven, such changing space weather makes a significant impact on the estimation of the drag coefficient. Based on the characteristics of the DTM94 atmospheric model and the test calculation on the satellite orbit, we adopt an empirical piecewise method in the evaluation process for the drag coefficient. If the solar radio activity is at a relative high level, manifested by the satisfaction of one of the following criteria (1) $\left|F_{10.7}-\bar{F}_{10.7}\right|>30$, (2) $\left|\bar{F}_{10.7}-150\right|>30$, a drag coefficient is estimated every half day, where $F_{10.7}$ represents the daily $10.7-\mathrm{cm}$ solar radio flux and $\bar{F}_{10.7}$ the 
arithmetic average of $F_{10.7}$ during the last three solar rotation periods. Alternatively, when the geomagnetic activity is relatively violent, which meets the condition $\left|\Delta\left[\Sigma K_{p}\right]\right|>100$, where $\Delta\left[\Sigma K_{p}\right]$ is the variation of intraday $\Sigma K_{p}$ (the sum of eight $K_{p}$ indices in one day) compared with that of the previous day, a drag coefficient is also estimated every half day. Otherwise, a drag coefficient is estimated every full day.

\section{Evaluation of orbit precision}

Except for the orbit arcs crossing the time of the maneuver, the precise orbit can be obtained with normal iterative convergence in the process of SLR dynamic short-arc orbit determination. Compared with those of the following months, the SLR tracking data in its first month, October 2011, had a relatively small volume, and in this month, there were some orbit adjustment processes which were not stated in detail. Thus our evaluation of orbit quality is carried out on the 6-month arcs from November 2011 to April 2012. Since no real satellite orbit can be obtained, the proper assessment of the precision of the SLR-derived orbit and the analysis of the orbital error are key issues after dynamic orbit determination. The present commonly-used evaluation methods on orbit precision include observational data fitting (internal accuracy), comparison between independent orbits (external accuracy), orbit overlap fitting, and station-satellite distance validation. In the following, the SLR orbit of HY-2 will be evaluated using three methods, which are SLR internal accuracy, orbit comparison between SLR and DORIS, and SLR ranging validation.

\subsection{SLR Internal accuracy}

The internal accuracy is often expressed in the form of the orbit-fitting root-mean-square (RMS) residual, which might be affected by some factors such as the quantities of and errors in measurement data, the orbit arc length, the number and piecewise method for estimated parameters, the level of data rejection, and the precision and convergence criteria of numerical integration [28]. The internal accuracies of HY-2 SLR orbits are represented in Figure 4. The starting time for every orbit arc is UTC 00:00. The average RMS value of 176 convergent orbits is $2.9 \mathrm{~cm}$, of which $75.6 \%$ are in the range from 2 to $4 \mathrm{~cm}$. As the orbits may partially deviate in the case of uneven distribution of the SLR tracking data, they should be evaluated by multi-type methods.

\subsection{Comparison with DORIS orbits}

The classical method of orbit comparison is to calculate the difference in the 3-D position and its components along the direction R/T/N (radial/transpose/normal) between two independent orbits [29]. Multi-techniques of orbit determination make HY-2 an ideal platform for tests on independent orbits. We will discuss the orbit comparison between the SLR- and DORIS-derived orbits.

DORIS is a precise Doppler tracking and radio-positioning system developed by the French CNES (Centre National d'Etudes Spatiales) [30]. The dual-frequency radio signals are transmitted in DORIS system under all-weather conditions. In comparison with SLR, there are more tracking stations (over 70) in the global DORIS network, and, with their more rational graphical distribution, low-orbit satellites can almost be tracked continuously. DORIS provides a method of high-precision orbit determination independent of SLR [31]. Currently, HY-2 is one of seven satellites supporting missions on tracking data and orbit service by the International DORIS Services (IDS) [32].

The time systems used in SLR orbits and DORIS-derived orbits, i.e. medium orbit ephemeris (MOE), are UTC (coordinated universal time) and TAI (international atomic time), respectively. At present, a constant offset (leap second) of $34 \mathrm{~s}$ is introduced in these two time scales (www.iers.org). Hence, initially the precise SLR orbits are interpolated by 9th-order Chebyshev polynomials to unify the nodetimes [33], and the mid-day orbits in the 3-day arc of SLR are then chosen to establish the comparison. Finally, taking the DORIS MOE as the reference orbit, the differences between these two orbits are calculated daily. As the thrust forces for the maneuver cannot be modeled precisely at present, the orbits at and shortly before and after the maneuver cannot

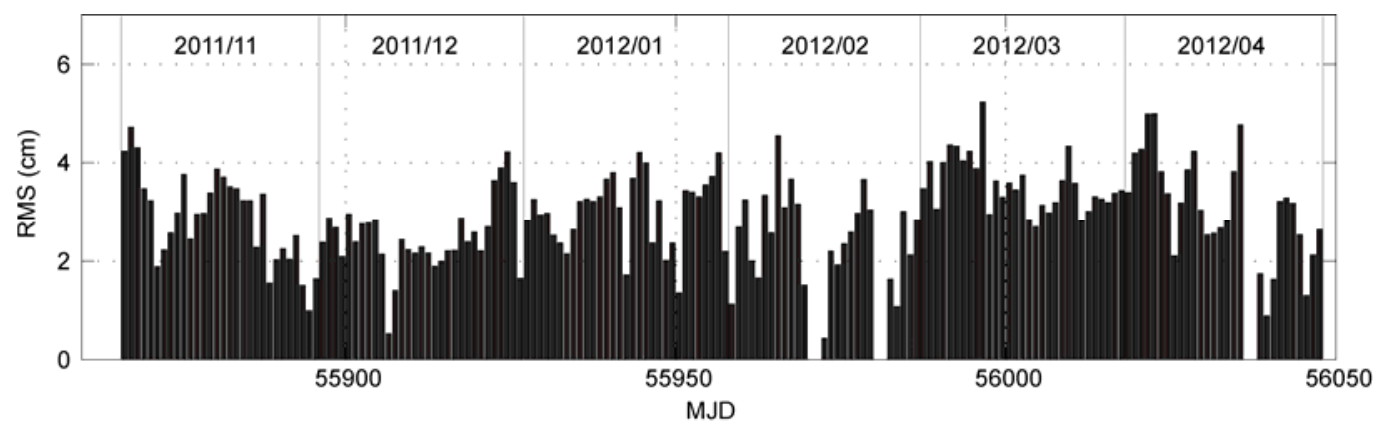

Figure 4 Internal accuracies of HY-2 SLR-derived orbits. 
be defined effectively using the dynamic method. As the last three maneuvers were performed from UTC 03:05 to UTC 03:20, the orbits were compared only on the segments from UTC 06:00 to UTC 24:00 for those three days.

In the processing of the evaluation, we find that when there were too few SLR trackings in a certain day, the differences between two orbits were significantly larger than those in the average case or when there were more SLR trackings. This is because too few observations provide poor constraints which are inadequate for orbit calculation. Thus, if the SLR tracking data on a certain day are less than 5 passes, we extend the length of the orbit arc for these days from 3 to 5 days, and after that, the related procedures are continued.

Figure 5 illustrates the 3-D position RMS differences between the SLR precise orbits and DORIS MOE, in centimeters, and their R/T/N components. Statistics show the 3-D RMS differences are less than $15 \mathrm{~cm}$ for $78.0 \%$ of the arcs, and less than $12 \mathrm{~cm}$ for $53.8 \%$ of the arcs. Figures 3 and 5 show that the especially large discrepancies (mainly along the $\mathrm{T}$ direction) in the time spanning four days around February 25, 2012 are the consequences of extremely sparse SLR tracking data on February 25 and 27, 2012. Table 2 lists the monthly average values along the $\mathrm{R} / \mathrm{T} / \mathrm{N}$ directions and the 3-D position RMS differences between the SLR orbits and DORIS MOE. The statistics in February 2012 did not include the corresponding values from 24th to 27th. It can be seen that the differences between them are generally
Table 2 Statistics on monthly average values of R/T/N and 3-D position RMS differences between SLR orbits and DORIS MOE

\begin{tabular}{lcccc}
\hline \multicolumn{1}{c}{ Time } & $\sigma_{3-\mathrm{D}}(\mathrm{cm})$ & $\sigma_{\mathrm{R}}(\mathrm{cm})$ & $\sigma_{\mathrm{T}}(\mathrm{cm})$ & $\sigma_{\mathrm{N}}(\mathrm{cm})$ \\
\hline Nov. 2011 & 10.07 & 2.37 & 7.81 & 5.63 \\
Dec. 2011 & 11.02 & 2.16 & 8.60 & 6.24 \\
Jan. 2012 & 12.18 & 2.43 & 8.45 & 8.14 \\
Feb. 2012 & 13.04 & 3.11 & 9.30 & 8.22 \\
Mar. 2012 & 11.54 & 2.67 & 8.52 & 6.93 \\
Apr. 2012 & 14.33 & 2.46 & 10.18 & 9.41 \\
Overall average & 12.03 & 2.53 & 8.81 & 7.43 \\
\hline
\end{tabular}

stable. It is obvious that the RMS differences along the R direction are smallest, with levels generally lower than $5 \mathrm{~cm}$ and an overall average of $2.53 \mathrm{~cm}$. The total average for the 3-D position RMS differences is $12.03 \mathrm{~cm}$ and the RMS difference along the $\mathrm{T}$ direction, with a total average of 8.81 $\mathrm{cm}$, is the worst of the three directions. The RMS difference along the $\mathrm{N}$ direction is $7.43 \mathrm{~cm}$ on average. These results coincide with the general characteristics of dynamic orbit determination, because the SLR tracking measurements are sensitive to radial positions, while insensitive to along-track positions of the satellite. Too few SLR trackings will lead to significant differences in all three directions.

Figure 6 demonstrates the time series of the residuals between the SLR orbits and DORIS MOE (every $60 \mathrm{~s}$, along the $\mathrm{R}, \mathrm{T}$ and $\mathrm{N}$ directions from up to down, in meter units during the week from March 12 to 18, 2012). The time
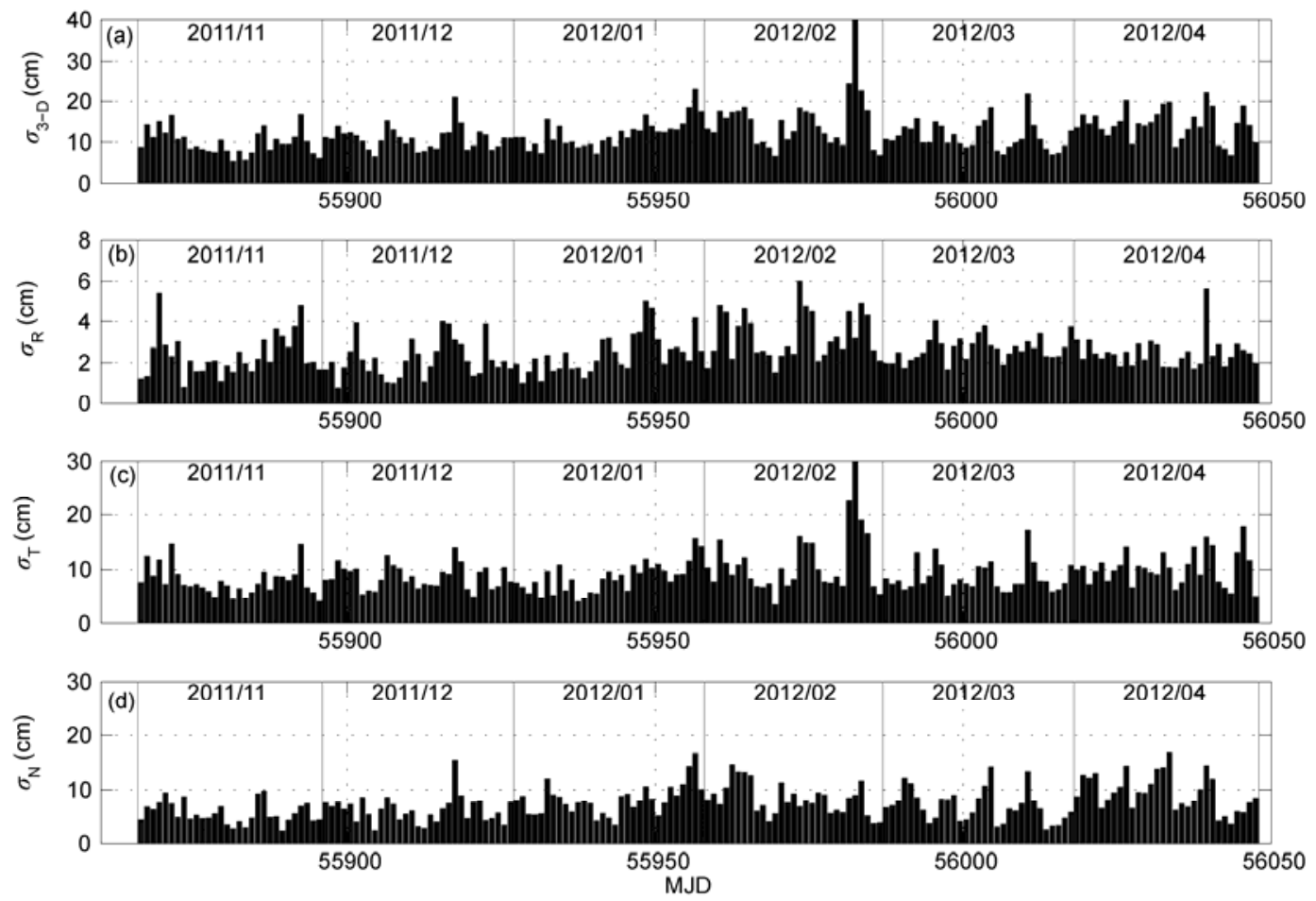

Figure 5 Daily RMS differences for 3-D positions between the SLR orbits and DORIS MOE (a), and its components along the direction R (b), T (c) and N (d). 

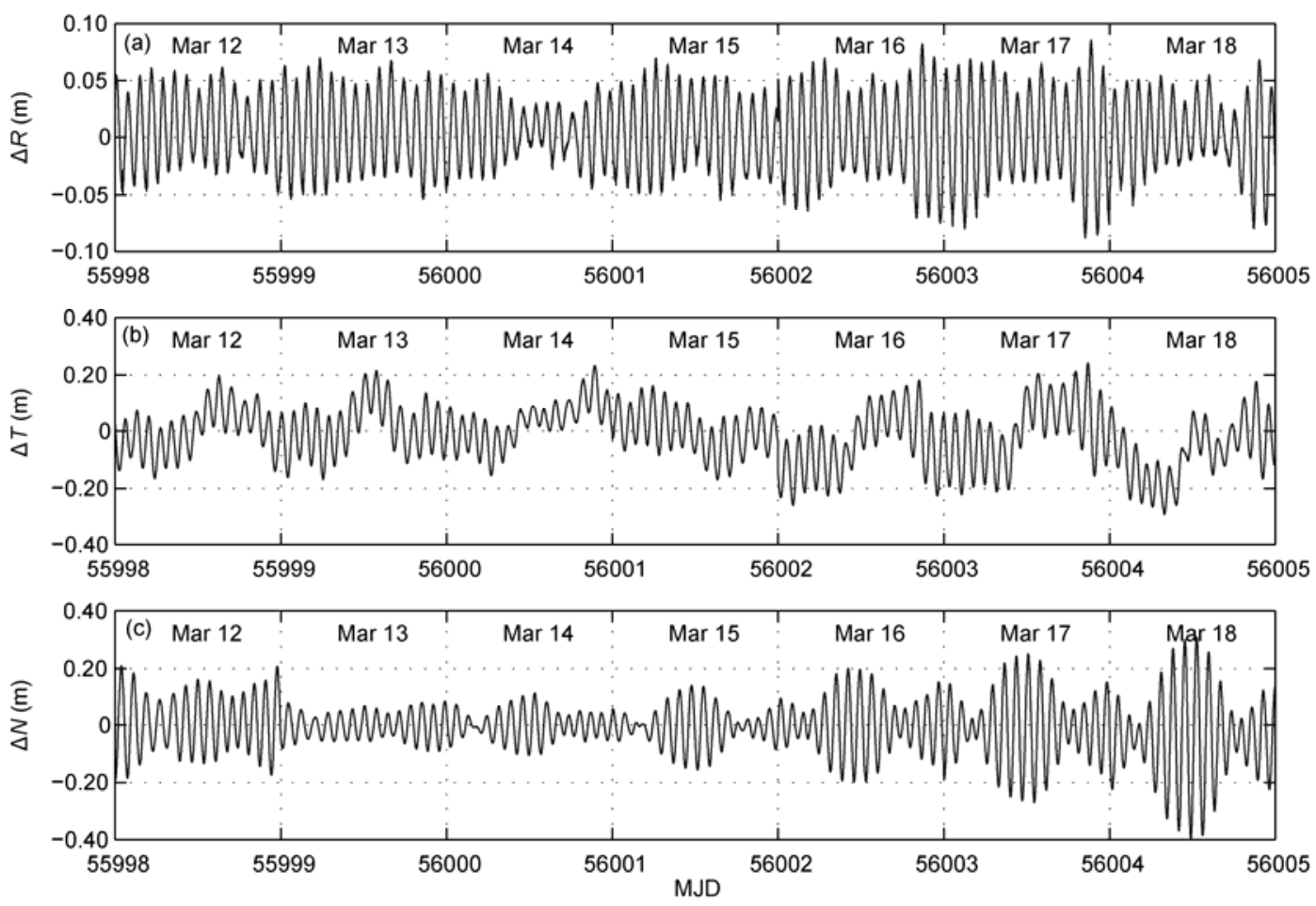

Figure 6 Differences every minute along the direction R (a), T (b) and N (c) between the SLR orbit and DORIS MOE in the week from March 12 to 18 , 2012.

interval between adjacent points was $60 \mathrm{~s}$. The SLR tracking data were distributed quite evenly during these days, 110 passes were tracked from 18 stations, and 1090 normal points were generated. The mean residuals along the $\mathrm{R} / \mathrm{T} / \mathrm{N}$ directions were $0.41 \pm 3.01 \mathrm{~cm},-0.83 \pm 8.83 \mathrm{~cm}$ and $-0.65 \pm$ $8.06 \mathrm{~cm}$, and the average 3-D RMS difference was 12.48 $\mathrm{cm}$. This again illustrates, the highest precision was along the $\mathrm{R}$ direction. Furthermore, no significant systematic biases existed between the SLR orbits and DORIS MOE. The differences mainly represent the models' errors relevant to the orbital period (about $1.7 \mathrm{~h}$ ) and the piecewise method adopted when estimating the atmospheric drag coefficient (the coefficient is estimated once per half day during this week).

\subsection{SLR distance validation}

Another important application of SLR is that it can validate orbits derived externally from other methods [34,35]. As the ranging precision of SLR is now at the centimeter level or even better, and with no influence from ambiguity and ionospheric delay, it can validate the result of DORIS orbit determination. The residuals obtained from the validation are the difference between the direct station-satellite distance from the SLR measurements and the derived station-satellite distance from DORIS MOE. The 9th-order Chebyshev interpolations [33] are implemented on DORIS MOE before the comparison because there is no one-to-one correspondence between the SLR ranging moments and the
MOE nodetimes. To extract radial errors from the residuals, the SLR data with an elevation cutoff of $60^{\circ}$ were chosen for the validation.

The validation results are shown in Figure 7, in which the $x$-coordinates represent the observation time, and $y$-coordinates represent the SLR RMS residual in centimeter units. A total of 1189 SLR ranging moments were extracted in the 6 months concerned. Further analysis demonstrated that the distance residuals between MOE and SLR were universally better than $6 \mathrm{~cm}$, and the statistics bias was $-0.65 \pm 3.31 \mathrm{~cm}$, which agreed with the result from the SLR-MOE orbit comparison. It can be concluded that no significant systematic biases existed between the SLR and MOE-derived distances. The radial precision for the SLR orbit was better than $4 \mathrm{~cm}$. Thus SLR can be taken as an efficient tool to evaluate the POD quality.

\section{Summary and conclusions}

We have summarized the latest SLR tracking data for HY-2, applied dynamic orbit determination, and calculated its precise orbits over six months. After the evaluations using SLR internal accuracy, comparisons between SLR and DORIS orbits, and the validation from station-satellite ranging, we can draw the following conclusions.

(1) Continuous and stable observation data on HY-2, including daylight and twilight time, can be obtained by the international SLR monitoring. On average, more than 10 


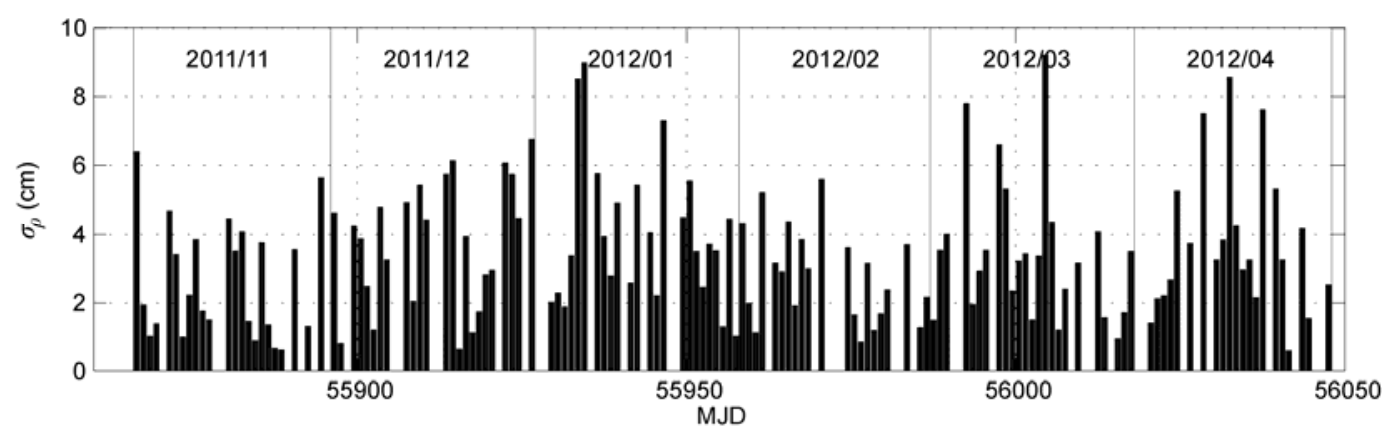

Figure 7 Ranging residual RMS between SLR and DORIS MOE.

passes can be observed and more than 100 normal points can be generated daily, which can provide effective constraints on the precise orbit determination of HY-2.

(2) Our models adopted in SLR orbit determination and the methods of SLR data processing are stable and reliable. Internal accuracy of the SLR orbit is around $3 \mathrm{~cm}$. No significant systemic biases have been found between the DORIS MOE and SLR orbits. The 3-D position precision for the SLR orbit is about $12.5 \mathrm{~cm}$ and the precision for the radial position is better than $3 \mathrm{~cm}$.

(3) No significant systemic biases have been found between SLR-measured station-satellite distances and DORISderived station-satellite distances. Statistical RMS differences between these two distances are better than $4 \mathrm{~cm}$ with an SLR elevation cutoff of $60^{\circ}$.

The international SLR monitoring on HY-2 is the first high-precision international SLR tracing carried out on a Chinese earth observation satellite. On the basis of close international collaboration over the past thirty years, the current Chinese SLR network can make full use of international monitoring resources to provide high-precision and high-reliability orbit service for HY-2 so as to satisfy the requirements of related scientific applications in precise satellite orbits.

The authors thank ILRS for providing the HY-2 SLR tracking data, and thank CNES for providing the HY-2 DORIS MOE. This work was supported by the National Natural Science Foundation of China (11173049, 11103064 and 11003036).

1 Liu J Q. Progress in and suggestion on the construction of Chinese sea satellite system and satellite marine application system (in Chinese). Adv Mar Sci, 2004, 22: 219-224

2 Lin M S. User requirements analysis of HY-2 (in Chinese). Sat Appl, 2003, 11: 7-13

3 Jiang X W, Lin M S, Liu J Q. Application prospects of HY-2 dynamic environment satellite (in Chinese). Sat Appl, 2011, 19: 4-8

4 Tapley B D, Ries J C, Davis G W, et al. Precision orbit determination for TOPEX/POSEIDON. J Geophys Res, 1994, 99: 24383-24404

5 Marshall J A, Zelensky N P, Klosko S M, et al. The temporal and spatial characteristics of TOPEX/POSEIDON radial orbit error. J Geophys Res, 1995, 100: 25331-25352

6 Bonnefond P, Exertier P, Schaeffer P, et al. Satellite altimetry from a short-arc orbit technique: Application to the Mediterranean. J Ge- ophys Res, 1995, 100: 25365-25382

7 Luthcke S B, Zelensky N P, Rowlands D D, et al. The 1-centimeter orbit: Jason-1 precision orbit determination using GPS, SLR, DORIS, and altimeter data. Mar Geod, 2003, 26: 399-421

8 Guo R, Hu X G, Tang B, et al. Precise orbit determination for geostationary satellites with multiple tracking techniques. Chin Sci Bull, 2010, 55: 687-692

9 Guo J Y, Han Y B. Seasonal and inter-annual variations of length of day and polar motion observed by SLR in 1993-2006. Chin Sci Bull, 2009, 54: 46-52

10 Yin Z Q, Han Y B, Podesta R, et al. South American SLR stations monitoring ground displacement caused by the M8.8 Chilean earthquake of 2010. Chin Sci Bull, 2011, 56: 738-742

11 Qu W J, Wu B. Analysis of the characteristics of the harmonics coefficient J2 of the Earth's gravity field in different periods. Chin Sci Bull, 2012, 57: 1626-1630

12 Wu B. ILRS SLR mission support request form: HY-2 retroreflector information. In: International Laser Ranging Services, 2011, 1-3

13 Gurtner W, Noomen R, Pearlman M R. The International Laser Ranging Service: Current status and future developments. Adv Space Res, 2005, 36: 327-332

14 Wu B, Lin M S, Zhang Z P. Global SLR tracking support for HY-2 satellite precise orbit determination. In: Proceedings of the 17 th international workshop on laser ranging, Bad Koetzting, Germany, 2011

15 Han Y B, Liu W D, Actis E, et al. Successful operation of a cooperative SLR station of China and Argentina in San Juan. Chin Sci Bull, 2008, 53: 2417-2420

16 Tapley B D. Fundamentals of orbit determination. In: Theory of Satellite Geodesy and Gravity Field Determination. New Work: Springer-Verlag, 1989. 235-260

17 Tapley B, Ries J, Bettadpur S, et al. GGM02-An improved Earth gravity field model from GRACE. J Geod, 2005, 79: 467-478

18 Standish E M. JPL Planetary and Lunar Ephemerides, DE405/LE405. JPL Interoffice Memorandum IOM 1998, 312.F-98-048

19 Berger $\mathrm{C}$, Biancale R, Ill M, et al. Improvement of the empirical thermospheric model DTM: DTM94 - A comparative review of various temporal variations and prospects in space geodesy applications. J Geod, 1998, 72: 161-178

20 Rim H J. TOPEX orbit determination using GPS tracking system. Ph.D. Thesis. Austin: University of Texas at Austin, 1992

21 McCarthy D D. Effect of solid earth tides. In: IERS Conventions 1996. IERS Technical Note, No. 21, 1996, 40-46

22 Eanes R, Schuler A. An improved global ocean tide model from TOPEX/POSEIDON altimetry: CSR4.0. In: EGS 24th General Assembly, Hague, Netherlands, 1999

23 McCarthy D D, Petit G. Equations of motion for an artificial earth satellite. In: IERS Conventions 2003. IERS Technical Note, No. 32, 2003, 106-107

24 Colombo O L. The dynamics of Global Positioning System orbits and the determination of precise ephemerides. J Geophys Res, 1989, 94: 9167-9182 
25 Marcos F A, Bowman B R, Sheehan R E. Accuracy of Earth's thermospheric neutral density models. In: AIAA/AAS Astrodynamics Specialist Conference, Keystone, CO, 2006, AIAA 2006-6167, 1-20

26 White O, Kopp G, Snow M, et al. The solar cycle 23-24 minimum. A benchmark in solar variability and effects in the heliosphere. Sol Phys, 2011, 274: 159-162

27 Pesnell W D. Solar cycle predictions. Sol Phys, 2012, doi: 10.1007/ s11207-012-9997-5

28 Tapley B D, Schutz B E, Born G H. The orbit problem, orbit accuracy. In: Statistical Orbit Determination. Burlington, MA: Elsevier Academic Press, 2004. 82-83

29 Montenbruck O, Holt G. Spaceborne GPS receiver performance testing. DLR-GSOC TN02-04. 2002, Deutsches Zentrum fur Luft- und Raumfahrt, Oberpfaffenhofen, 1-41

30 Tavernier G, Fagard H, Feissel-Vernier M, et al. The International DORIS Service: Genesis and early achievements. J Geod, 2006, 80:
403-417

31 Barlier F. The DORIS system: A fully operational tracking system to get orbit determination at centimeter accuracy in support of Earth observations. Comptes Rendus Geosci, 2005, 337: 1223-1224

32 Ferrage P. GGOS Bureau for Networks and Communications. International Doris System report 2011. GGOS meetings, San Francisco, CA, 2011

33 Sagovac C P, Danielson D A, Clynch J R, et al. Fast interpolation for Global Positioning System (GPS) satellite orbits. NPS-MA-95-006, Technical Report Naval Postgraduate School, Monterey, CA, 1995, $1-28$

34 Barlier F, Berger P, Bonnefond P, et al. Laser-based validation of GLONASS orbits by short-arc technique. J Geod, 2001, 75: 600-612

35 Peng D J, Wu B. Percise orbit determination for Jason-1 satellite using on-board GPS data with cm-level accuracy. Chin Sci Bull, 2009, 54: 196-202

Open Access This article is distributed under the terms of the Creative Commons Attribution License which permits any use, distribution, and reproduction in any medium, provided the original author(s) and source are credited. 